\title{
Characterization of Cell Cycle Phase-Based microRNAs in Pluripotency and Differentiation
}

\author{
Xiaoyan Ming, Timothy Ming-Hun Wan, Lin Chen, Maria Mercedes Garcia-Barcelo, Chung Mau Lo and Xiao Qi Wang* \\ Department of Surgery, The University of Hong Kong, Hong Kong, China
}

\begin{abstract}
In addition to signaling pathways, transcription factors and epigenetic regulators, microRNAs (miRNAs) are emerging as important regulators of human pluripotent stem cells (hPSCs). Pluripotent miRNAs that regulate G1-S transition and pluripotency factors to maintain self-renewal have been identified. However, only 4-5 clusters of miRNAs have been identified in human embryonic stem cells (hESCs). We performed cell cycle phase-based (G1, S and G2/M phases) miRNA array in pluripotent and differentiated hESCs. We demonstrated that embryonic stem cell-cell cycle (ESCC) regulating miRNAs were all highly expressed in three cell cycle phases of undifferentiated hESCs suggesting a non-cell phase regulated mechanism. From cell phase-dependent miRNAs, G2/M-miRNAs was extracted by principle component analysis (PCA) as a significant component in pluripotent hESCs, whereas G1-miRNAs was a significant component in differentiated hESCs. The results indicate that G2/M-miRNAs might function to maintain pluripotency and G1-miRNAs might function to enhance differentiation. By miRNA target site prediction, G2/M-phase miRNA displayed potential target sites on differentiation factors GATA6 and GATA4, G1-phase miRNAs displayed potential target sites on pluripotency gene OCT4, NANOG and SOX2, which warrant further confirmation and functional study. By statistical and computation analysis of the miRNA array data, we demonstrated that the G2/M-miRNAs could potentially repress differentiation factors to maintain pluripotency and G1-miRNAs could potentially target pluripotency genes to enhance differentiation.
\end{abstract}

Keywords: microRNAs; Cell cycle phases; Pluripotency; Differentiation

\section{Introduction}

Pluripotent stem cells (PSCs) are defined by their capacity to undergo indefinite self-renewal and to differentiate into all somatic cell lineages and germ cells [1], which includes embryonic stem cells (ESCs) and induced pluripotent stem cells (iPSCs). PSCs have unique cell cycle characteristics; cycling cells have a short G1 phase and a large percentage of $S$ phase cells, whereas upon induction of differentiation the G1 phase lengthens and the fraction of cells in S phase is reduced [2]. G1 cells are prone to differentiation signaling cues, such as retinoic acid (RA), while $S$ phase cells are refractory to this RA signal [3]. More importantly, the transcript levels of developmental genes have been found to be cell cycle regulated, peaking in G1 and declining in S phase, respectively [4]. Thus, G1 is the cell fate decision-making point; when pluripotent cells enter into G1, they become "poised" and permissive to either continued self-renewal or commitment to lineage specification [4].

Small non-coding RNAs, the microRNA (miRNA) superfamily has been revealed as a new class of stem cell lineage determiners. Like transcription factors, a single miRNA can regulate the target gene products. Among miRNAs expressed in ESCs, the conserved miR$302 / 367$ cluster is highly expressed and has the most functional impact. Another less conserved miRNA cluster for ESCs is the miR-290-292 cluster in mESCs and miR-371-373 cluster in mEpiSCs/hESCs. Dicer and Dgcr are required for the processing of mature miRNA. Dicer and Dgcr8 knockout mESCs and hESCs exhibit proliferation and cell cycle G1-S transition defects, whereas miR-302 and miR-290 clusters can partially rescue these defects. Thus, these miRNAs are also called embryonic stem cell-cell cycle regulating (ESCC) miRNAs. All these miRNAs are highly expressed in undifferentiated ESCs and decline upon differentiation, which indicates a specific function for these miRNAs in ESCs [5-12].

During differentiation, ESCs shift to alternative molecular programs to inhibit self-renewal and coordinate differentiation into highly specialized cell types. A direct way to silence the self-renewal is to repress the pluripotency transcription factors such as OCT4, SOX2 and NANOG [8]. MiR-145 specifically binds the 3'UTR of OCT4 to repress pluripotency in hESCs. MiR-145 also directly regulates SOX2 and KLF4 [13]. Another group of pluripotency-silencing miRNAs are the let-7 family, which are widely expressed in various somatic tissues. Let-7 is repressed in ESCs but rapidly upregulated during ESC differentiation. The let-7 family directly targets hundreds of members of the pluripotency network including c-Myc, Sall4, and $\operatorname{Lin} 28$ to induce differentiation [14]. On the other hand, the ESCC miRNAs indirectly upregulate many of these same let-7 targets [14]. Although exactly how the ESCC miRNAs are functionally dominant over let-7 remains unknown; that the ESCC miRNAs can upregulate Lin28, which negatively regulates the maturation of the let-7 family of miRNAs is likely part of the mechanism [5,9]. Thus, the ESCC miRNAs and several differentiation-inducing miRNAs provide contrasting mechanisms to monitor the cell cycle and self-renewal of ESCs [5].

Pluripotent miRNAs that are highly expressed in ESCs have been identified. These miRNAs regulate G1-S transition and directly or indirectly regulate pluripotency factors to maintain the self-renewal. However, only 4-5 clusters of either pluripotency maintaining miRNAs or pluripotency-silencing miRNAs have been identified in hESCs. In our present study, we performed cell cycle phase-based (G1, S and G2/M phases) miRNA array on pluripotent and differentiated hESCs, Heatmap and principal components analysis (PCA) to organize and visualize miRNA array data, and PCA-SPSS to extract common factors

*Corresponding author: Xiao Qi Wang, D.Phil. Department of Surgery, The University of Hong Kong, 21 Sassoon Road, Hong Kong, Tel: 852-39179653; Fax: 852-39179634; E-mail: xqwang@hku.hk

Received December 31, 2016; Accepted January 18, 2017; Published January 25, 2017

Citation: Ming X, Ming-Hun Wan T, Chen L, Garcia-Barcelo MM, Lo CM, et al. (2017) Characterization of Cell Cycle Phase-Based microRNAs in Pluripotency and Differentiation. Cell Dev Biol 6: 178. doi:10.4172/2168-9296.1000178

Copyright: ( 2017 Ming X, et al. This is an open-access article distributed under the terms of the Creative Commons Attribution License, which permits unrestricted use, distribution, and reproduction in any medium, provided the original author and source are credited. 
among cell-phase miRNA variables. We demonstrated cell cyclephase specific miRNAs. The G2/M-miRNAs could potentially repress differentiation factors to maintain pluripotency and G1-miRNAs could potentially target pluripotency genes to enhance differentiation.

\section{Materials and Methods}

\section{Cell culture}

The hESC line H1 was maintained in feeder-free mTeSR1 or E8 medium (Stem Cell Technology, Vancouver, Canada) on Matrigel (BD Biosciences, San Jose, CA, USA) or Geltrex (Invitrogen, Carlsbad, CA USA) at $37^{\circ} \mathrm{C}$ in a humidified atmosphere of $5 \% \mathrm{CO}_{2}$. Medium was changed daily with daily. $2-5 \mu \mathrm{M}$ of retinoic acid (Sigma-Aldrich, St. Louis, MO, USA) was added to hESCs for 2-3 days for in vitro differentiation [15].

\section{Flow cytometry and cell sorting}

To obtain cell cycle-based cell fractions, $\mathrm{H} 1$ cells in pluripotency and differentiated states were harvested and fixed in cold $80 \%$ ethanol followed by staining with propidium iodide (PI) for cell cycle analysis as described previously [16,17]. G1, S and G2/M cells were sorted out based on DNA content using BD FACSAria I Cell Sorter (BD Biosciences). Sorted G1, S and G2/M cell pellets were frozen immediately at $-70^{\circ} \mathrm{C}$ for RNA extraction.

\section{RNA extraction and miRNA cDNAs reverse transcription}

Total RNA was extracted using RNAiso Plus (total RNA extraction reagent, Takara, Shiga, Japan), a representative Trizol reagent. Total RNA was suspended in Invitrogen UltraPure DNase/RNase-free distilled water (Invitrogen). The RNA concentrations were determined using NanoDrop ND-1000 spectrophotometer (Nanodrop Technologies, Wilmington, DE, USA).

\section{miRNAs cDNAs reverse transcription}

$800 \mathrm{ng}$ of total RNA from each cell phase was applied to reverse transcribe mRNA to complementary DNA (cDNA) using Megaplex reverse transcription (RT) primers and TaqMan microRNA RT kit (Applied Biosystems, Foster City, CA, USA) following manufacturer's instructions. Megaplex primers enhance the ability to detect low expression of miRNAs and TaqMan only quantitate specific mature miRNAs, not precursors.

\section{TaqMan human microRNA array analysis}

Synthesized miRNA cDNA were applied to quantify miRNAs expression using the TaqMan ${ }^{\circ}$ Array Human microRNA A and B Cards Set v2.0 (Applied Biosystems). Loading of the A and B cards and running the arrays on the $7900 \mathrm{H}$ fast real-time PCR system followed the manufacturer's instructions. The miRNA array $\mathrm{A}+\mathrm{B}$ card sets cover 667 of human miRNAs. Ct values were used to present miRNA expression levels. For differential miRNA expression amongst cell cycle phases, $\Delta \Delta \mathrm{Ct}$ was calculated as fold change versus cell phase with the lowest level of miRNA.

\section{Statistical analysis}

A web tool ClustVis [18] was used for HeatMap and PCA to organize and visualize miRNA array data. SPSS 21 (IBM Corp., Armonk, NY, USA) was applied to perform PCA. Bartlett's test sphericity with an associated $\mathrm{p}$ value was $<0.001$ before further valid factor analysis. Rotated component matrix was used to interpret the component.

\section{Results}

\section{Cell cycle phase-based ESCC miRNA profile}

Undifferentiated and differentiated cycling hESC line $\mathrm{H} 1$ cells were sorted by flow cytometry into G1, S and G2/M cohorts, respectively, according to DNA content (Figure 1A). Cell cycle phase-based miRNA profiles were detected using TaqMan human microRNA array analysis. ESCC miRNAs are pluripotent miRNAs regulating G1-S transition, although it is not known whether ESCC miRNAs function in cell phase-based pattern. We firstly characterized ESCC miRNA levels in each cell cycle. ESCC miRNA expression levels can clearly separate pluripotency and differentiated hESC population according to PCA (Figure 1B), with ESCC miRNAs were highly expressed in pluripotent hESC cells (Figure 1C, left panel), whereas significantly down-regulated during differentiation (Figure $1 \mathrm{C}$, right panel). We also noticed that the ESCC or ESCC-related miRNA, such as miR-302-367 cluster, were highly expressed in all phases of the cell cycle (Figure 1C). Moreover, in pluripotency states, ESCC miRNAs of respective G1, S and G2/M cell phase were located closely together or overlapped in the PCA map, but clearly separated after differentiation (Figure 1B). The data indicates that most known ESCC miRNAs were independent of cell cycle phase-based expression manner to maintain pluripotency in hESCs, whereas ESCC miRNAs became more differentially expressed in different cell cycle in differentiated hESCs. Thus, although ESCC miRNAs accelerated G1-S transition, their expressions are not cell cycle-phase regulated.

\section{Cell cycle phase-based miRNA profile}

We next characterized miRNA profile from all cell phase-based miRNA expression array data. To observe the relationship between pluripotent and differentiated hESCs, PCA analysis clearly separated pluripotent and differentiated hESC population. However, G1-, Sor G2/M-based miRNAs were not integrated but rather separated in distance in pluripotent hESCs (Figure 2A), indicating that miRNAs of each cell phase were differentially expressed, which was a distinctive pattern comparing to G1-, S- and G2/M-ESCC miRNAs that were ubiquitously expressed (Figure 1B). HeatMap analysis further highlighted some miRNAs were cell cycle-phase differentially expressed in both pluripotent and differentiated hESCs (Figures 2B and 2C). Unlike ESCC miRNAs, which were not cell cycle-phase regulated (Figure 1C), this part of the results suggested that there were miRNAs that were expressed in a cell cycle phase-dependent manner.

\section{G2/M-miRNAs was a significant component in pluripotent hESCs}

To further characterize the miRNAs that are differentially expressed in different phases of the cell cycle, PCA-SPSS was applied to determine the possibility of making a predictive model. From miRNA variables among G1, S, and G2/M, the extracted component 1 comprised of G2/ M-miRNAs (Figures 3A and 3B) and explained $48.9 \%$ of the variation; another component 1 comprised of G1-miRNAs and explained $33.8 \%$ of the variation. Thus, G2/M miRNAs could be a common variance among G1, S, and G2/M variables in pluripotent hESCs. In differentiated hESCs, levels and expression pattern of miRNAs in the G1, S and G2/M were altered with overall decreased miRNA expression including G2/M-miRNAs (Figure 2C). PCA-SPSS analysis extracted G1 miRNAs as component 1 (Figures 3C and 3D) and explained $57.36 \%$ of the variation. To examine the interrelation among a set of variables of G1, S and G2/M miRNAs and extracted significant component 1 from different cell-phase miRNAs in pluripotent versus differentiated hESCs, we demonstrated that G2/M-miRNAs were a statistically 
A

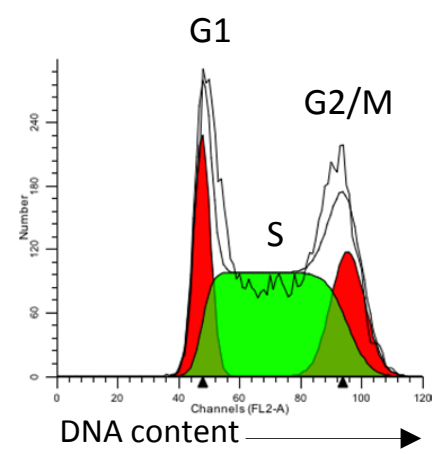

B

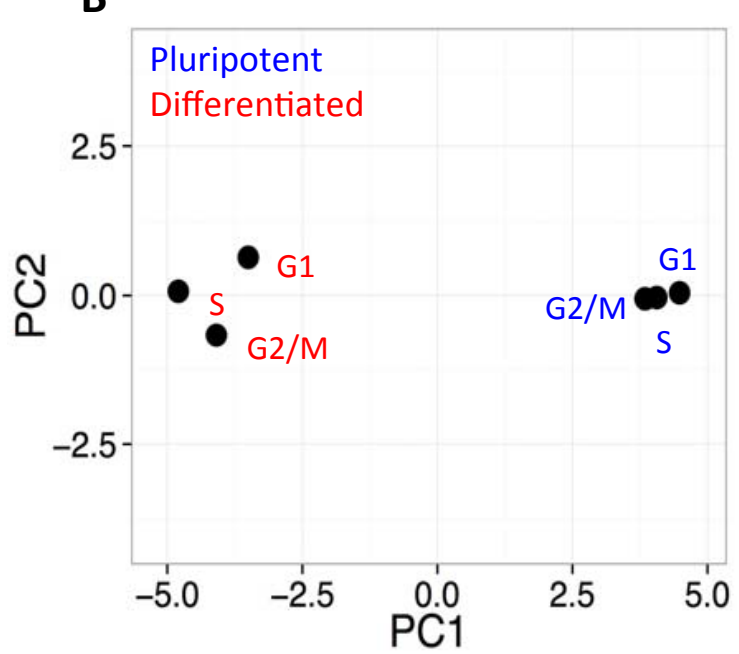

C

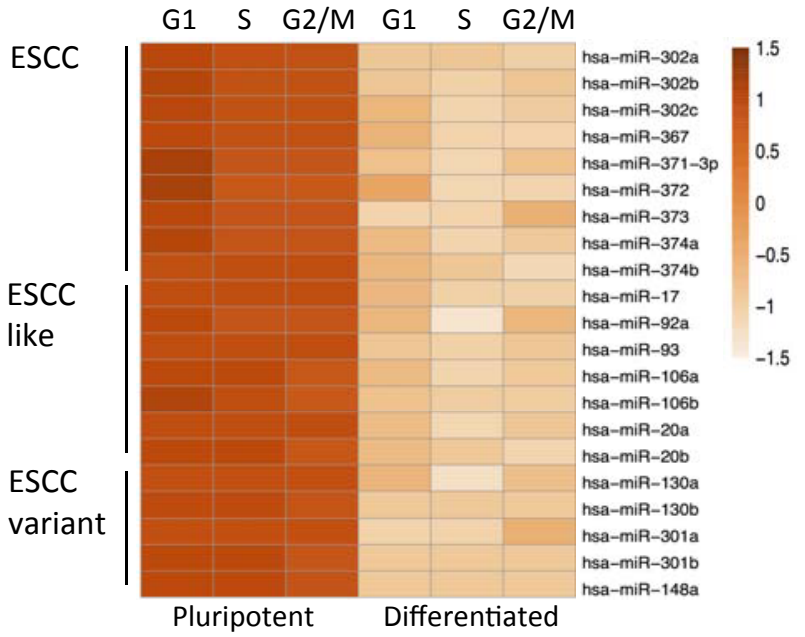

Figure 1: Cell cycle phase-based ESCC miRNAs profile. (A) Histogram of DNA content of hESCs. G1, S and G2/M cells were sorted out respectively based on the histogram of DNA content. (B) PCA pattern to separate ESCC miRNAs between pluripotent and differentiated hESCs. (C) HeatMap to show expression pattern of ESCC (including ESCC, ESCC-like and ESCC-variant) miRNAs in G1, S and G2/M cell cycle phases, respectively, in undifferentiated and differentiated hESCs. ESCC miRNA were highly expressed in pluripotent hESCs and down regulated in differentiated hESCs in a cell cycle-independent manner.

significant component among G1, S and G2/M miRNA variables in pluripotent state, whereas G1-miRNAs were a significantly component in differentiation state. The results suggested that G2/M-miRNAs in pluripotency and G1-miRNAs in differentiation might contain regulatory messages for pluripotency and differentiation, respectively.

\section{G2/M-miRNAs consist of miRNAs that target differentiation factors}

Given that G2/M-miRNAs was a statistically significant component in pluripotent hESCs whereas G1-miRNAs became a significant component after hESCs differentiation, we hypothesized that in pluripotent state G2/M-miRNAs may comprise of miRNAs to maintain pluripotency. In differentiation state, G1-miRNA may comprise of miRNAs to enhance differentiation. We therefore searched the miRNAs that were expressed in either G1 or G2/M phase in undifferentiated hESCs but silenced in differentiated hESCs; alternatively, miRNAs that were silenced in $\mathrm{G} 1$ or G2/M phase in undifferentiated hESCs but expressed in G1 or G2/M phase (Supplementary Table 1). Potentially, miRNAs expressed in undifferentiated but silenced in differentiation states might function to repress differentiation genes (GATA4, GATA6, EOMES, GSC and MIXL1) for pluripotency. To search for potential targets of the negative regulation by these miRNAs, we used platforms of microRNA.org, Targetscan and miRDB.

Among 22 miRNAs that expressed at G2/M phase in undifferentiated hESCs but silenced in differentiated hESCs (Supplementary Table 1), 13 out of 22 (59\%) displayed potential target sites on GATA6 (Table 1), 6 out of $22(27 \%)$ target sites on GATA4, and 3 out of 22 (13.6\%) target sites on GSC (data not shown). These G2/M phase-based miRNAs had few target sites on EOMES and MIXL1. Among microRNA/mRNA prediction methods, miRanda-mirSVR is a regression model for predicting the likelihood of target mRNA down-regulation based on sequence and structure features, which identifies a significant number of experimentally determined non-canonical and non-conserved sites. It ranks miRNA target sites by down-regulation score, with the suggested mirSVR score cutoff being -0.1 or lower $[19,20]$ (http://www. microRNA.org). We further detected that 8 out of $22 \mathrm{G} 2 / \mathrm{M}$-miRNAs (36.4\%) targeting GATA6 with mirSVR lower than -0.1 , ranging from -0.11 to -1.348 (Table 1 ). On GATA6 mRNA, miR-181c, miR-624-5p 
A

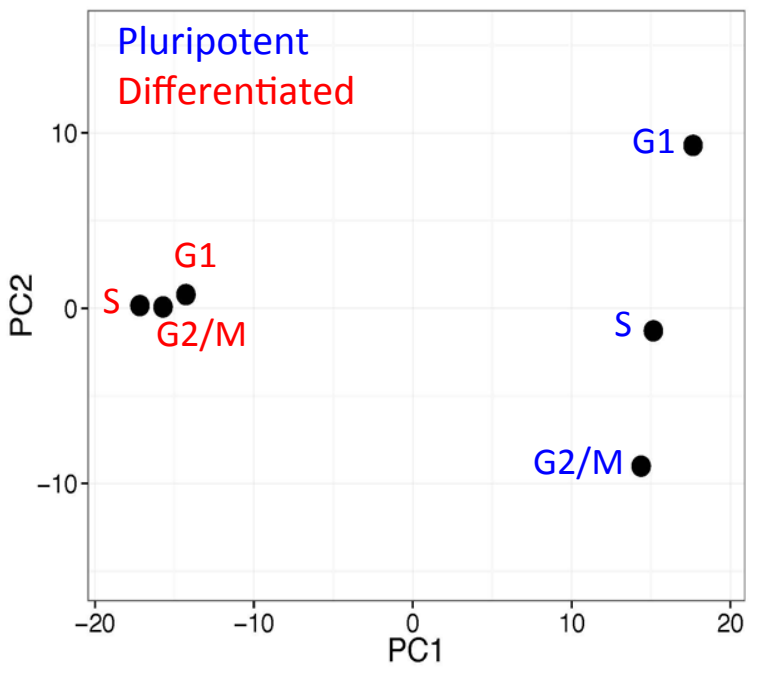

B

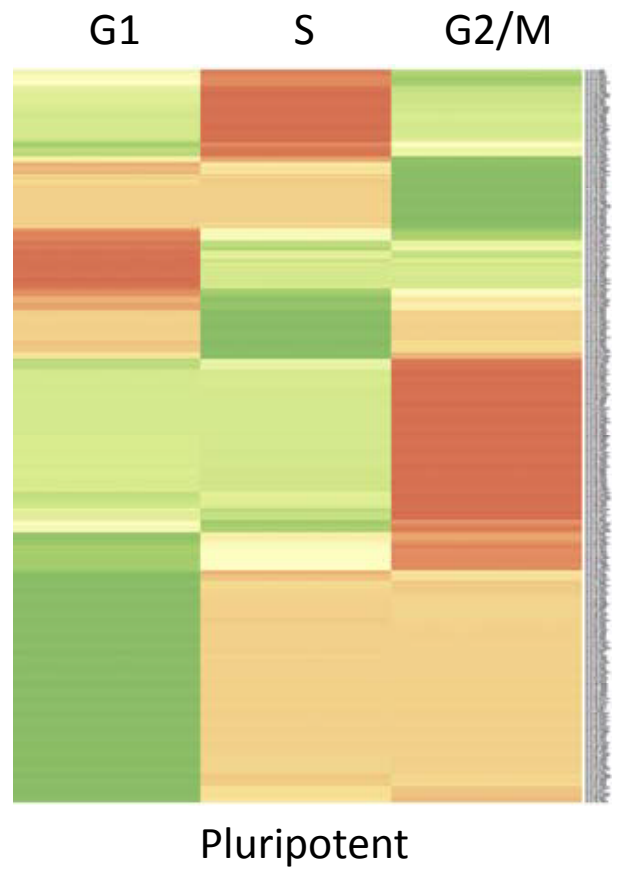

C

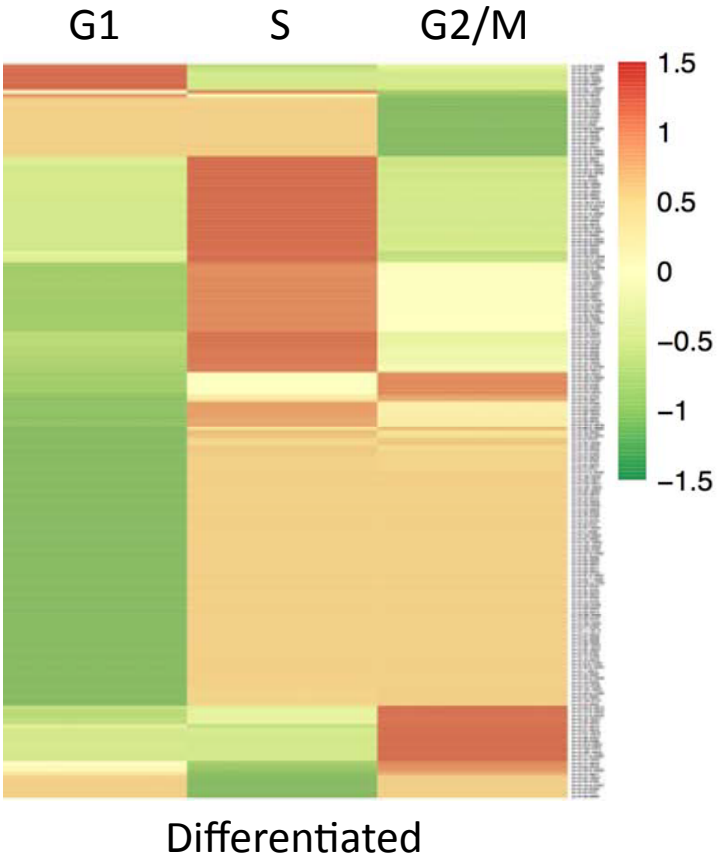

Figure 2: Cell cycle phase-based miRNA array profile. (A) PCA pattern based on miRNAs array data of cell-phase miRNAs in undifferentiated and differentiated hESCs, where the expression of G1-, S- and G2/M-miRNAs in undifferentiated hESCs was distant. (B) HeatMap of G1-, S- and G2/M-miRNAs in undifferentiated hESCs. (C) HeatMap of G1-, S- and G2/M-miRNAs in differentiated hESCs.

\begin{tabular}{|c|c|c|c|}
\hline $\begin{array}{c}\text { \# of G2/M-miRNAs (Expressed in pluripotency and } \\
\text { silenced in differentiation) }\end{array}$ & $\begin{array}{c}\text { \% of G2/M-miRNAs (Target sites on } \\
\text { GATA6) }\end{array}$ & $\begin{array}{c}\text { mirSVR score* } \\
<-0.1\end{array}$ & $\begin{array}{c}\text { mirSVR score } \\
<-1\end{array}$ \\
\hline 22 & $59 \%(13 / 22)$ & $36.4 \%(8 / 22)$ \\
\hline
\end{tabular}

${ }^{*}$ miSVR score is an algorithm method for ranking microRNA target sites by down-regulation score

Table 1: Predicted GATA6 target sites of G2/M-miRNAs.

and miR-367-5p showed mirSVR score of target sites $-1.3481,-1.3421$ and -1.0286 , respectively. These mirSVR scores indicated that these miRNA sites were expected to be functional sites (Figures 4A-4C). On the other hand, only $14.3 \%$ of G1-phase miRNAs (expressed at G1 phase in undifferentiated hESCs but silenced in differentiated hESCs) showed target sites on GATA6 with mirSVR score lower than -0.1 , ranging from -0.111 to -0.649 . Thus, compared to G2-phase miRNAs, G1-phase miRNAs were less likely functional.

G1-miRNAs of differentiated hESCs displayed miRNAs targeting pluripotency genes

Given that G1-miRNAs were a significant component in 
A

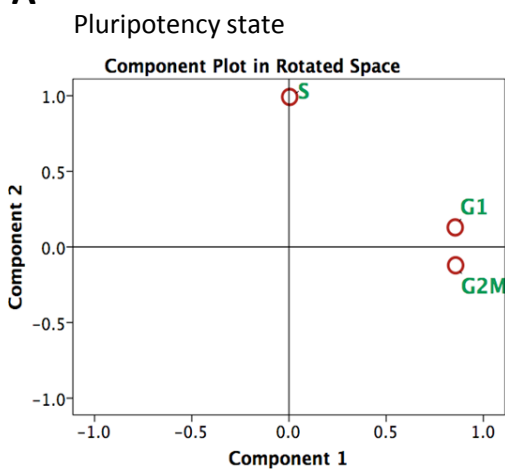

C

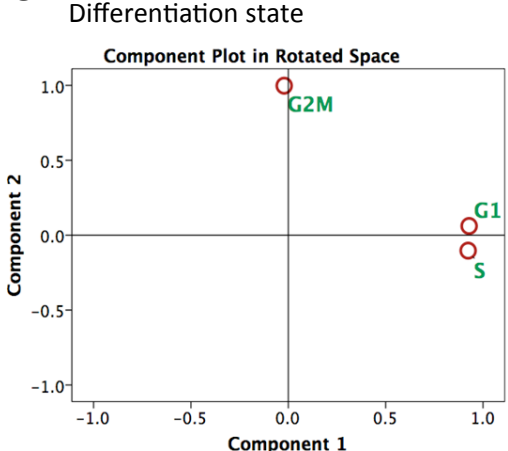

B

Rotated component matrix

\begin{tabular}{|l|r|r|}
\hline \multirow{2}{*}{} & \multicolumn{2}{|c|}{ Component } \\
\cline { 2 - 3 } & 1 & \multicolumn{1}{c|}{2} \\
\hline G2/M & .856 & -.122 \\
G1 & .854 & .130 \\
S & & .992 \\
\hline
\end{tabular}

D

Rotated component matrix

\begin{tabular}{|l|r|r|}
\hline \multirow{2}{*}{} & \multicolumn{2}{|c|}{ Component } \\
\cline { 2 - 3 } & 1 & \multicolumn{1}{c|}{2} \\
\hline G1 & .929 & \\
S & .924 & -.103 \\
G2/M & & .998 \\
\hline
\end{tabular}

Figure 3: Principle component extraction of cell phase variable miRNAs by PCA-SPSS. (A) and (B) Cell cycle phase differentially expressed miRNAs were subjected to PCA-SPSS analysis. Although Kaiser-Meyer-Olkin (KMO) Measure of Sampling Adequacy (MCA) was low (0.48), Bartlett's test sphericity associated p value was $<0.001$ for a valid factor analysis. PCA-SPSS pattern and rotated component matrix showed that G2/M-miRNAs was significant component 1 in pluripotent hESCs. (C) and (D) PCA-SPSS pattern and rotated component matrix showed that G1-miRNA was significant component 1 in differentiated hESCs.

A GATA6 5' agcauuuuuuauaaUGAAUGUa 3'
miR-181c 3' ugaguggcuguccaACUUACAa 5'
GATA6 5' uaAACAUUUUAACUUAAUGGUACUu 3'
miR-624-5p 3' acUUGU-----GUUCCAUGACCAUGAu 5'
mirSVR score: -1.3481

PhastCons score: 0.8395

mirSVR score: -1.3421

PhastCons score: 0.8135

mirSVR score: -1.0286

PhastCons score: 0.8395

Figure 4: Predicted target sites of G2/M-miRNAs miR-181c, miR-624-5p and miR-367-5p on GATA6 3'UTR sequences. (A) Interaction between miR-181c and the target site annotated on the 3'UTR of GATA6 and miSVR score prediction by microRNA.org. (B) Interaction between miR-624-5p and target site on the 3'UTR of GATA6. (C) Interaction between miR-367-5p and target site on the 3'UTR of GATA6.

differentiation state (Figures $3 \mathrm{C}$ and $3 \mathrm{D}$ ), we next determined the G1-mRNAs that were silenced in pluripotent state but expressed after differentiation. From miRNA array profile, 12 miRNAs were from silence in pluripotency to expression in G1 in differentiation; 2 miRNAs were from silence in pluripotency to expression in G2/M in differentiation (Supplementary Table 1). We hypothesized that expressed G1-miRNAs during differentiation may function to repress pluripotency genes. To explore the potential target mRNAs by G1-miRNAs, we found that pluripotency genes OCT4, NANOG, and SOX2 all contained target sites of G2-miRNA miR-361-3p and miR-186-3p, with Context++ percentile 88-95 (TargetScanHuman 7.1). KLF4 contained target sites of miR-623 and miR-124. These results suggest that miRNAs that only expressed in G1 phase during differentiation might potentially function to regulate pluripotency genes negatively.

\section{Discussion}

We established cell cycle-based (G1, S and G2/M phases) miRNA data set from pluripotent and differentiated hESCs by human miRNA array. As expected, ESCC, ESCC-like and ESCC-variant miRNAs were highly expressed in pluripotent hESCs and significantly down regulated in RA-induced differentiation of hESCs. However, most of ESCC and ESCC-related miRNAs showed the pattern of non-cell cycle-regulated. Mouse ESCC miRNAs are known to target many inhibitors of the G1/S transition including Cdkn1a (p21), Lats2 and Rb1 [8]. More than promoting the G1/S transition by targeting CDKN1A, some of human ESCC miRNAs target WEE1 (a G2/M checkpoint kinase), Cdk2Cyclin D and Cdk4-Cyclin D complex, which result in promoting both G2/M and G1/S transition [8]. Moreover, the transcription of ESCC 
miRNAs is regulated by pluripotency factors such as OCT4, SOX2 and NANOG [8]. Thus, even their expression in non-cell cycle phase-based pattern, ESCC miRNAs control proliferation, survival, cell cycle and pluripotency program of pluripotent stem cells.

PCA-SPSS is a statistical technique to examine the interrelations among a set of variables in order to identify the underlying structure of variables. Extracted 'principle components' account for most of the variance in the original variables. It is a non-parametric analysis and the result is unique and independent of any hypothesis for data distribution. Using PCA-SPSS to analyse differentially expressed miRNAs of G1-, S- and G2/M-cell cycle phase in both pluripotent and differentiated hESCs, G2/M-miRNAs were extracted as statistically significant component 1 in undifferentiated hESCs. Interestingly, in differentiated hESCs, G1-miRNAs were significant component 1, whereas G2/M-miRNAs was no longer a significant component. The clear shift of significant component 1 of cell-phase miRNAs between pluripotency and differentiation suggested that G2/M-miRNAs was mostly down-regulated after differentiation.

The cell cycle machinery and its associated signaling pathways play critical roles in regulation of stem cell properties. First is the critical link between G1 length and differentiation, when pluripotent cells enter into G1, they become "poised" and permissive to either continued selfrenewal or commitment to lineage specification [21]. Secondly, recent study has shown that the $S$ and G2 phases are intrinsically associated with maintenance of pluripotency, where suppression of regulators of the S and G2 phases leading to a rapid decrease in expression of pluripotency markers [22,23]. Thus, proposed novel mechanistic model is that cell fate specification starts in the G1 phase where hESCs can sense differentiation signals; then cell fate commitment is completed in $\mathrm{G} 2 / \mathrm{M}$, where pluripotency is dissolved and differentiation completed via cell cycle-dependent mechanisms $[22,23]$. Of miRNA array analysis of pluripotent and differentiated hESCs, we identified that cell phasebased miRNAs displayed distinct features in different hESC states: G2/ M-miRNAs is a significant component in pluripotent hESCs, but G1miRNAs is a significant component in differentiated hESCs. Seemingly, these characteristics of cell phase-based miRNAs are in accordance with pluripotency regulation through cell cycle-dependent mechanisms. Moreover, by statistical and computation analysis, we demonstrated that G2/M-miRNAs in pluripotent state might comprise miRNAs to maintain pluripotency and G1-miRNAs in differentiation state might comprise miRNAs to enhance differentiation. The results indicated a strong link between bioinformatics and biological understanding potential.

By searching for potential targets for the negative regulation of cell phase-based miRNAs, we demonstrated that some G2/M-miRNAs of pluripotent hESCs had potential target sites on differentiation factors such as GATA6 and GATA4 and G1-miRNAs of differentiation hESCs had potential target sites on pluripotency gene OCT4, NANOG, SOX2 and KLF4. More importantly, 36.4\% of G2/M-phase miRNAs comprising target sites on GATA6 were highly possible being functional. Therefore, some of cell-phase miRNAs might have potentials to be novel miRNAs to regulate pluripotency genes or differentiation factors for hESC self-renewal and differentiation, which worth investigations of specific miRNA binding sites confirmation and further functional study. In summary, our cell cycle phase-miRNA array measurement and bioinformatics analysis demonstrated that the G2/M-miRNAs could potentially target differentiation factors to maintain pluripotency and G1-miRNAs could potentially target pluripotency genes to enhance differentiation (Figure 5).

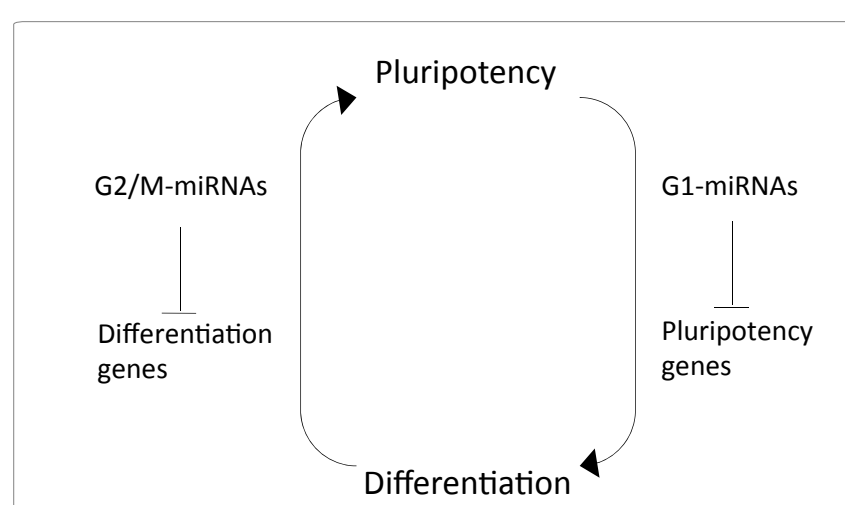

Figure 5: Schematic figure to illustrate the potential of G2/M-miRNAs (in undifferentiated hESCs) could target differentiation factors for maintainence of pluripotency and G1-miRNAs (in differentiated hESCs) could target pluripotency genes to enhance differentiation.

\section{Conclusion}

MicroRNAs are one of the important regulators of hPSCs, although only 5 clusters of either pluripotency-maintaining miRNAs or pluripotency-silencing miRNAs have been identified in hESCs. Through statistical and computational analysis of cell cycle-based miRNA arrays, we identified G2/M-miRNAs might comprise of miRNAs that target differentiation factors and G1-miRNAs might comprise of miRNAs that target pluripotency genes. Therefore, from a bioinformatic point of view, our study provides novel information on cell phase-based miRNAs which regulate pluripotency and differentiation.

\section{Acknowledgement}

This work was partially supported by Health and Medical Research Fund Research Council of Hong Kong to XQ Wang (HMRF 03143396).

\section{Disclosure}

Authors declare that they have no conflict interest regarding the publications of this paper.

\section{References}

1. Smith AG (2001) Embryo-derived stem cells: Of mice and men. Annu Rev Cell Dev Biol 17: 435-462.

2. Singh AM, Dalton S (2009) The cell cycle and Myc intersect with mechanisms that regulate pluripotency and reprogramming. Cell Stem Cell 5: 141-149.

3. Mummery CL, van Rooijen MA, van den Brink SE, de Laat SW (1987) Cell cycle analysis during retinoic acid induced differentiation of a human embryonal carcinoma-derived cell line. Cell Differ 20: 153-160.

4. Singh AM (2015) Cell cycle-driven heterogeneity: On the road to demystifying the transitions between "poised" and "restricted" pluripotent cell states. Stem Cells Int 2015: 219514

5. Greve TS, Judson RL, Blelloch R (2013) microRNA control of mouse and human pluripotent stem cell behavior. Annu Rev Cell Dev Biol 29: 213-239.

6. Ivey KN, Srivastava D (2010) MicroRNAs as regulators of differentiation and cell fate decisions. Cell Stem Cell 7: 36-41.

7. Rosa A, Brivanlou AH (2013) Regulatory non-coding RNAs in pluripotent stem cells. Int J Mol Sci 14: 14346-14373.

8. Guo W-T, Wang XW, Wang Y (2014) Micro-management of pluripotent stem cells. Protein Cell 5: 36-47.

9. Gangaraju VK, Lin H (2009) MicroRNAs: Key regulators of stem cells. Nat Rev Mol Cell Biol 10: 116-125.

10. Martinez NJ, Gregory RI (2010) MicroRNA gene regulatory pathways in the establishment and maintenance of ESC identity. Cell Stem Cell 7: 31-35

11. Wang Y, Baskerville S, Shenoy A, Babiarz JE, Baehner L, et al. (2008) Embryonic stem cell-specific microRNAs regulates the G1-S transition and promote rapid proliferation. Nat Genet 40: 1478-1483. 
Citation: Ming X, Ming-Hun Wan T, Chen L, Garcia-Barcelo MM, Lo CM, et al. (2017) Characterization of Cell Cycle Phase-Based microRNAs in Pluripotency and Differentiation. Cell Dev Biol 6: 178. doi:10.4172/2168-9296.1000178

12. Wang Y, Melton C, Li YP, Shenoy A, Zhang XX, et al. (2013) miR-294/miR302 promotes proliferation, suppresses G1-S restriction point, and inhibits ESC differentiation through separable mechanisms. Cell Rep 4: 99-109.

13. Xu N, Papagiannakopoulos T, Pan G, Thomson JA, Kosik KS (2009) MicroRNA-145 regulates OCT4, SOX2 and KLF4 and represses pluripotency in human embryonic stem cells. Cell 137: 647-658.

14. Melton C, Judson RL, Blelloch R (2010) Opposing microRNA families regulate self-renewal in mouse embryonic stem cells. Nature 463: 621-626.

15. Wang XQ, Lo CM, Chen L, Ngan ES, Xu A, et al. (2016) CDK1-PDK1-PI3K/Akt signaling pathway regulates embryonic and induced pluripotency. Cell Death Differ [Epub ahead of print]

16. Wang XQ, Zhu YQ, Lui KS, Cai Q, Lu P, Poon RT (2008) Aberrant Polo-like kinase 1-Cdc25A pathway in metastatic hepatocellular carcinoma. Clin Cancer Res 14: 6813-6820

17. Wang X, Lui VC, Poon RT, Lu P, Poon RY (2009) DNA damage mediated s and $g(2)$ checkpoints in human embryonal carcinoma cells. Stem Cells $27: 568-576$
18. Metsalu T, Vilo J (2015) ClustVis: A web tool for visualizing clustering of multivariate data using principal component analysis and Heatmap. Nucleic Acids Res 43: W566-W570.

19. Betel D, Koppal A, Agius P, Sander C, Leslie C (2010) Comprehensive modeling of microRNA targets predicts functional non-conserved and non-canonical sites. Genome Biol 11: R90.

20. Betel D, Wilson M, Gabow A, Marks DS, Sander C (2008) The microRNA.org resource: Targets and expression. Nucleic Acids Res 36(Database Issue) D149-D153.

21. Gonzales KA, Liang H, Lim YS, Chan YS, Yeo JC, et al. (2015) Deterministic restriction on pluripotent state dissolution by cell-cycle pathways. Cell 162: 564579 .

22. Vallier $L$ (2015) Cell cycle rules pluripotency. Cell Stem Cell 17: 131-132

23. Wang XQ, Stanbridge EJ (2015) Cell cycle and cell cycle regulators in the process of development, pluripotency, differentiation and reprogramming. Frontiers in Stem Cell and Regenerative Medicine Research 2: 176-189.
Citation: Ming X, Ming-Hun Wan T, Chen L, Garcia-Barcelo MM, Lo CM, et al. (2017) Characterization of Cell Cycle Phase-Based microRNAs in Pluripotency and Differentiation. Cell Dev Biol 6: 178. doi:10.4172/2168-9296.1000178
OMICS International: Open Access Publication Benefits \& Features

Unique features:

Ulncreased global visibility of articles through worldwide distribution and indexing

Showcasing recent research output in a timely and updated manner

Special issues on the current trends of scientific research

Special features:

$700+$ Open Access Journals

$50,000+$ editorial team

Rapid review process

Quality and quick editorial, review and publication processing

Indexing at major indexing services

Sharing Option: Social Networking Enabled

- Authors, Reviewers and Editors rewarded with online Scientific Credits

Better discount for your subsequent articles

Submit your manuscript at: http://www.omicsonline.org/submission 